Original Article

\title{
Evaluation of the effects of different treatments for the elbow joint using joint proprioception and surface electromyography
}

\author{
Qiuchen Huang, PT, PhD ${ }^{1,2)}$, Kuei-Yuan $\mathrm{WANG}^{3)}$, Lili Yu, $\mathrm{PT}^{1,2)}$, Yue Zhou, PT ${ }^{1,2)}$, \\ Rui Gu, PhD ${ }^{1,4)}$, Yao Cui, PT ${ }^{1,2)}$, Chunying Hu, PT ${ }^{1,2)^{*}}$, Meng Ge, PT ${ }^{5)}$, Yanfeng Xu, PT $^{5}$, \\ JIANFENG LIU, PT ${ }^{5}$ \\ 1) School of Rehabilitation Medicine, Capital Medical University, China \\ 2) Department of Physical Therapy, China Rehabilitation Research Center: No.10, North Road, Fengtai \\ District, Beijing 100077, China \\ 3) National Taiwan Sport University, Taiwan \\ 4) Department of Orthopedic and Orthopedic Rehabilitation, China Rehabilitation Research Center, \\ China \\ 5) Rehabilitation Therapy Department, PKU Care Rehabilitation Hospital, China
}

\begin{abstract}
Purpose] The purpose of this study was to examine the immediate effects of strength training and neuromuscular joint facilitation (NJF) distal resistance training on muscle strength and proprioception. [Subjects] The subjects were 15 young healthy people $(29.3 \pm 4.1 \mathrm{y}, 166.8 \pm 7.1 \mathrm{~cm}, 62.4 \pm 11.6 \mathrm{ky})$. [Methods] Two isometric contraction techniques were applied on the elbow joint: elbow joint flexion muscle strength training (MST) and the elbow joint flexion pattern of NJF. Muscle strength (measured by surface electromyography [sEMG]) and joint position errors of the left upper limb were measured before and after one intervention session of MST and NJF. [Results] The decrease in error in elbow flexion angle repetition represented the improvement resulting from NJF. sEMG of the biceps brachii showed significant increases in the maximum discharge and average discharge after the intervention. [Conclusion] This result suggests that elbow joint proprioception and muscle strength can be improved by NJF together with proximal resistance training.

Key words: Neuromuscular joint facilitation, Joint position error, Elbow joint proprioception
\end{abstract}

(This article was submitted Aug. 4, 2015, and was accepted Sep. 25, 2015)

\section{INTRODUCTION}

Patients with cervical spinal cord injury often have impaired proprioception of the upper limb and reduced muscle strength and range of movement (ROM). Proprioception training, muscle strength training (MST), and ROM training are usually implemented in physical therapy programs for patients with cervical spinal cord injury ${ }^{1}$. A number of research studies have focused on increasing muscle strength and/or ROM of the upper limb. After cervical spinal cord injury, patients' upper limb proprioception is evaluated to know the extent of the disability and its effect on the activities of daily living, and has also been used as a part of treatment for cervical spinal cord injury as well as practical functional treatment in occupational therapy rehabilitation programs.

Along with muscle strength, proprioception sense is

*Corresponding author. Qiuchen Huang (E-mail:

qiuchen_1984@126.com)

(C2015 The Society of Physical Therapy Science. Published by IPEC Inc. This is an open-access article distributed under the terms of the Creative Commons Attribution Non-Commercial No Derivatives (by-ncnd) License $<$ http://creativecommons.org/licenses/by-nc-nd/3.0/> also decreased in patients with cervical spinal cord injury. The joint position error (JPE) test is considered the primary measure of upper limb proprioception and has been widely used as an outcome indicator for patients with cervical spinal cord injury ${ }^{2}$. A decrease in JPE indicates increased ability to reposition the joint after active movement. In clinical treatment and research, resistance to elbow joint flexion is used to enhance the strength of the biceps brachii, whereas in clinical treatment, isotonic contraction is used. Evaluation of proprioception is not performed to assess or compare other manipulation therapies.

Neuromuscular joint facilitation (NJF) is a new therapeutic exercise based on kinesiology that integrates the facilitation element of proprioceptive neuromuscular facilitation (PNF) and joint composition movement, aiming to improve the movement of the joint through passive, active, and resistance exercises ${ }^{3)}$. NJF is used to increase strength, flexibility, and $\mathrm{ROM}^{4}$, and improve elbow function. NJF uses the same motion pattern as PNF, but the location of resistance of NJF is different, i.e., proximal resistance is applied to the biceps or to the brachialis muscle tendon attachment point in elbow patterns.

The purpose of this study was to examine the immediate effects of strength training and NJF distal resistance training 
using surface electromyography (sEMG) and evaluation of proprioception using the JPE test.

\section{SUBJECTS AND METHODS}

Subjects were 15 young healthy people ( 3 males and 5 females). Subjects' characteristics are detailed in Table 1 . The purpose and contents of this research were explained to the subjects, and they gave their informed consent to participate in the study. The study was approved by the Research Ethics Committee of China Rehabilitation Research Center (IRB no. 2014-26).

The subjects sat on a chair and placed their left upper limb on the table in front. The subjects' shoulder joint was flexed at $90^{\circ}$ and the elbow joint was fully extended. Two isometric contractions were performed on the elbow joint: elbow joint flexion MST and the elbow joint flexion pattern of NJF. All interventions were carried out by one physiotherapist.

1. MST group: One hand of the examiner was placed against the distal forearm. The other hand of the examiner was placed on the distal humerus to fixate the elbow joint. Resistance was applied as the subjects performed elbow joint flexion.

2. NJF group: The elbow flexion-forearm supination (FFS) pattern and elbow flexion-forearm pronation (FFP) pattern of NJF were performed. One hand of the examiner was placed against the distal forearm and traction and resistance were increased, as was done for the MST group. In the FFP pattern, the examiner's other hand is on the bicipital tubercle, which is the tendon attachment point of the biceps brachii. In the FFS pattern, the examiner's other hand is on the ulnar tuberosity, which is the tendon attachment point of the brachial muscle. When the subjects performed the elbow joint flexion pattern, traction and resistance were applied throughout the process by two hands.

In both, MST and NJF groups, the interventions were

Table 1. Subject characteristics

\begin{tabular}{lc}
\hline & $\mathrm{M} \pm \mathrm{SD} \mathrm{N}=24$ \\
\hline Age (yrs) & $29.3 \pm 4.1$ \\
Height $(\mathrm{cm})$ & $166.8 \pm 7.1$ \\
Weight $(\mathrm{kg})$ & $62.4 \pm 11.6$ \\
\hline
\end{tabular}

carried out six times. In the NJF, traction and resistance were applied three times on the bicipital tubercle and three times on the ulnar tuberosity ${ }^{5}$. There was a 1-hour rest period between interventions in the MST and NJF groups, and two interventions and tests were performed within 1 day. All trials were performed at random. Before and after one intervention of MST and NJF, JPEs were measured and sEMG was performed.

In the JPE test, the ROM measurement equipment (BioVal 4.51, SyCoMoRe 8.51, RMIngenierie, France) was used. The fixed and mobile arms were the vertical axis of the humerus and the radius; the flexion angle of elbow joint was recorded by the computer. The subjects were asked to close their eyes, and the examiner flexed the subject's elbow joint at a random angle. The subjects' elbow joint was then fully extended, and they were asked to flex the elbow at approximately the same angle as in the initial flexion. The error in the elbow flexion angle was recorded. Each measurement was carried out five times, and the average value was used for analysis. In the sEMG evaluation of the biceps brachii, maximum isometric contraction in the start position was maintained for 5 seconds, during which the maximum discharge and average discharge of the muscle were measured by an sEMG system (Telemyo 2400T; Noraxon, Scottsdale, AZ, USA).

Two-way repeated-measures analysis of variance (ANOVA) was used to test for statistically significant differences, and the factors were intervention and group. If significant interaction was found, paired t-test was performed to compare the outcome indicators before and after the intervention. Data were analyzed using SPSS Ver. 17.0 for Windows (SPSS, Chicago, IL, USA). The level of statistical significant was set at 0.05 .

\section{RESULTS}

Two-way ANOVA revealed significant interactions among the JPEs of the two groups, indicating that the changes between the groups were significantly different (Table 2). The error in the elbow flexion angle repetition was reduced by NJF intervention.

Two-way ANOVA showed the main effects of the intervention. The paired t-test showed significant increases in the maximum discharge and average discharge of the biceps

Table 2. Intervention effects of different treatments for the elbow joint on JPE as well as the maximum and average discharge as seen on sEMG

\begin{tabular}{|c|c|c|c|}
\hline & $\begin{array}{l}\text { Error of elbow flexion } \\
\text { angle }\left(^{\circ}\right)\end{array}$ & $\begin{array}{c}\text { Maximum discharge on } \\
\operatorname{sEMG}(\mu \mathrm{V})\end{array}$ & $\begin{array}{l}\text { Average discharge on } \\
\text { sEMG }(\mu \mathrm{V})\end{array}$ \\
\hline \multicolumn{4}{|l|}{ MST group } \\
\hline a. Before & $6.9 \pm 1.2$ & $2,607.4 \pm 1,229.6$ & $14,367.0 \pm 7,235.0$ \\
\hline b. After & $7.3 \pm 1.7$ & $3,127.0 \pm 1,472.2$ & $15,468.4 \pm 6,794.8$ \\
\hline \multicolumn{4}{|l|}{ NJF group } \\
\hline c. Before & $6.5 \pm 1.7$ & $3,171.5 \pm 1,243.7$ & $15,574.8 \pm 6,154.9$ \\
\hline d. After & $2.5 \pm 1.0$ & $3,400.7 \pm 1,208.1$ & $19,374.0 \pm 6,856.0$ \\
\hline
\end{tabular}


brachii on sEMG after the intervention. However, there was no significant difference between the MST group and the NJF group.

\section{DISCUSSION}

Compared with that of the MST group, the NJF group's error in elbow flexion angle repetition was reduced, and the maximum discharge and average discharge of the biceps brachii on sEMG were significantly increased after two treatments.

These results can be attributed to the improvement in the functions of the periarticular muscles of the elbow joint due to the application of distal resistance. Distal resistance and the approximation of the facilitation elements were included in both the groups; therefore, the maximum discharge and average discharge of the biceps brachii on sEMG increased in both MST and NJF groups.

The alignment of the elbow joint capsule, the functions of the periarticular muscle of the elbow joint, and the elbow position sense were improved; therefore, JPE decreased in the NJF group. In the NJF resistance exercise, the tendon of the biceps brachii and the brachial muscle were drawn out using proximal resistance on the bicipital tubercle and ulnar tuberosity ${ }^{6}$.

These results suggest that elbow joint proprioception and muscle strength can be improved by NJF together with proximal resistance training, which can be used as a new form of exercise for improving the function of the elbow joint.

\section{REFERENCES}

1) Treleaven J, Jull G, Sterling M: Dizziness and unsteadiness following whiplash injury: characteristic features and relationship with cervical joint position error. J Rehabil Med, 2003, 35: 36-43. [Medline] [CrossRef]

2) Chen $X$, Treleaven J: The effect of neck torsion on joint position error in subjects with chronic neck pain. Man Ther, 2013, 18: 562-567. [Medline] [CrossRef]

3) Huang Q, Li D, Yokotsuka N, et al.: The intervention effects of different treatment for chronic low back pain as assessed by the cross-sectional area of the multifidus muscle. J Phys Ther Sci, 2013, 25: 811-813. [Medline] [CrossRef]

4) Huo M, Maruyama H, Kaneko T, et al.: The immediate effect of lumbar spine patterns of neuromuscular joint facilitation in young amateur baseball players. J Phys Ther Sci, 2013, 25: 1523-1524. [Medline] [CrossRef]

5) Huo M: Neuromuscular Joint Facilitation. Tokyo: Ipec Press, 2010, p 76.

6) Wang H, Huo M, Huang Q, et al.: The immediate effect of neuromuscular joint facilitation (NJF) treatment on hip muscle strength. J Phys Ther Sci, 2013, 25: 1455-1457. [Medline] [CrossRef] 